\title{
Une approche psychomécanique du mot espagnol que
}

\author{
Samuel Bidaud ${ }^{*}$ \\ Université de Bourgogne, Francia
}

\section{INTRODUCTION}

Nous nous proposons dans cette note d'étudier dans ses grandes lignes le sémantisme du morphème espagnol que à partir d'une perspective psychomécanique. Après avoir rappelé le cadre général de cette théorie, nous nous proposerons de reconstruire l'idéogénèse de queet de voir à quel mouvement de pensée correspond ce mot.

\section{LA THEORIE PSYCHOMECANIQUE : QUELQUES PRINCIPES}

Il convient de rappeler brièvement quelques principes de linguistique guillaumienne sur lesquels se fonde notre approche de que.

Le premier principe est clair et a été rappelé à de nombreuses reprises par Gustave Guillaume: la psychomécanique est une linguistique mentaliste qui se doit de rechercher derrière les données que l'on peut observer en discours

* Para correspondencia, dirigirse a: Samuel Bidaud (samuel.bidaud@aliceadsl.fr), 1, rue Ernest Petit, 21000 Dijon, Côte d'Or, France/Francia. 
une unité cohérente et systématique de laquelle découle cedernier. C'est ce que formule Gustave Guillaume fréquemment: "toujours et partout, [...] découvrir le psychique sous le sémiologique" (Guillaume 1971a : 77), et ce

car la réalité, pour un bon observateur, ne se limite pas à ce qui tombe sous le coup de l'observation directe, par l'effet d'une existence sensible, et dispense ainsi l'esprit de tout effort tendant à une construction de l'objet à observer. La réalité, la vraie réalité, si j'ose dire, s'étend fort au-delà, et il n'est permis de la connaître qu'à ceux qui acceptent le risque de chercher, d'imaginer sous les faits visibles, constatables directement, des faits profonds et en quelque sorte secrets, qui, plus que les faits visibles, observables dans l'immédiat, sont la réalité (Guillaume 1971b : 10).

Le but du linguiste est donc d'arriver à un "voir de compréhension" (Guillaume 1973a)qui dépasse la pure observation des faits.

C'est dans le langage que se reflète la pensée qui se construit:

La psycho-systématique n'étudie pas les rapports de la langue et de la pensée, mais les mécanismes définis et construits que possède la pensée pour opérer une saisie d'elle-même, mécanismes dont la langue offre une reproduction fidèle. (Guillaume1973a : 94).

Or, ce que le linguiste doit comprendre, c'est non seulement le système de puissance, qui est situé au niveau de la langue, mais également la transition de ce système au discours, laquelle nécessite un temps infime mais réel néanmoins, nommé par Gustave Guillaume le temps opératif.

Ce temps peut être décomposé en deux mouvements de pensée : un mouvement de singularisation, qui va discerner dans la masse du pensable une idée, et un mouvement d'universalisation, qui va reverser cette idée à l'une des parties du discours. Le premier mouvement correspond à l'idéogénèse ; le second mouvement à la morphogénèse. Alors que l'idéogénèse permet d'aboutir à une idée singularisée, la morphogénèse donne à cette idée particularisée les caractéristiques formelles d'une partie $\mathrm{du}$ discours (genre, nombre, etc).

Soit le mot : livre. Ce mot est d'abord isolé dans la masse du pensable ; puis il se voit attribuer une forme, celle de substantif, pourvue d'un genre, masculin, d'un nombre, singulier, et d'une fonction.

Ce que l'on peut représenter sous la forme d'un tenseur binaire cher à Gustave Guillaume: 
(1) Construction du mot:

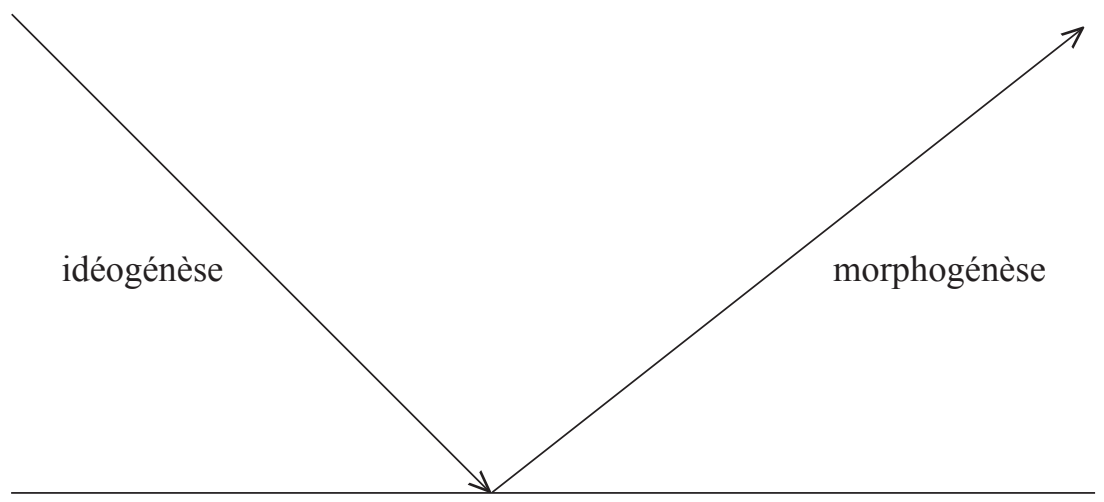

La pensée a donc besoin d'un temps opératif pour construire son signifié.

La théorie psychomécanique émet une hypothèse forte au sujet de ce dernier:

du côté psychique, $[\ldots]$ nous ne nous trouvons pas en présence de signifiants qui peuvent être théoriquement aussi divers, aussi hétérogènes que l'on voudra, du moment qu'ils signifient suffisamment, mais en présencedu signifié qui, lui, doit, dans la partie formelle de la langue, être aussi un que possible, et donc avoir acquis la cohérence systématique. (Guillaume 1973a : 129)

Gustave Guillaume nomme signifié de puissance «l' "invariant", la valeur fondamentale d'une forme» (Boone et Joly1996: 383), et ce signifié de puissance permet l'ensemble des effets de sens observés en discours.Le linguiste doit donc chercher la cohérence derrière l'incohérence que peut présenter le discours, comme c'est le cas avec l'imparfait, souvent commenté par Gustave Guillaume.

Le signifié de puissance se construit durant l'idéogénèse et est de nature cinétique. Les arrêts sur l'idéogénèse correspondent à des saisies. Chaque saisie peut être plus ou moins subduite par rapport au sens plein de l'idéogénèse (la subduction est un processus d'abstraction sémantique par rapport à ce sens plein). C'est ce que Gustave Guillaume a commenté à de nombreuses reprises, notamment avec les verbes être et avoir(Guillaume 1973b).

Nous verrons dans les pages qui suivent quelle représentation de langue nous pouvons donner du mot espagnol que, mot grammatical au cinétisme 
très développé qui n'a pas encore, sauf erreur de notre part, fait l'objetd'une approche psychomécanique.

\section{LE MOT ESPAGNOL QUE}

Si le mot que n'a pas encore été analysé dans une perspective psychomécanique pour l'espagnol, en revanche il l'a largement été pour le français et, dans une certaine mesure, pour l'italien (Moignet 1974). Récemment encore, Olivier Soutet(2005)a repris le problème pour développer une théorie de l'idéogénèse du mot français que tout à fait particulière.

Nous pouvons repartir de l'analyse qui nous est proposée du que français et du signifié de puissance qu'a dégagé Gérard Moignet, signifié qui correspond à un mouvement de l'actuel au virtuel :

cette tension est porteuse d'un processus de dématérialisation sémantique progressive, à partir de la représentation mentale d' «être inanimé virtuel». [...] On peut symboliser ce mouvement par un tenseur [...] au départ duquel figure (première saisie $\mathrm{S} 1$ ) la représentation mentale en question, et sur la progression duquel diverses étapes (S2, S3, S4, Sn...) marquent divers degrés successifs de dématérialisation, jusqu'à aboutir à un point où toute représentation d'être est abolie et où il n'en subsiste qu'un résidu extrêmement ténu (Moignet 1974 : 224).

Or, que ce soit pour le français ou pour l'espagnol, c'est un mouvement contraire que nous proposons, qui va du virtuel à l'actuel et qui se caractérise par un remplissage de matière sémantique. Arriver à l'actuel présuppose en effet d'être déjà passé par les états plus virtuels.

Reconstituons ce signifié de puissance.

Nous trouvons au départ de l'idéogénèse un que qui a pour seule fonction de poser la phrase qui le suit. Que intègre une phrase mais n'est pas intégré par elle et est une sorte d'appui emphatique qui pourrait être supprimé et laisser un contenu presque identique à la phrase. Appelons ce premier que emphatique. On le retrouve dans les phrases suivantes:

Pues la verdad es que estoy en la universidad más que nada porque, claro, es lo que hace todo el mundo después del instituto, que no te lo planteas y simplemente y directamente te metes en la universidad. (oral, d'après le Crea).

Lola: Venga papa, que te acompaño a la puerta... 
El padre de Lola: Bueno, encantado... si necesitáis una cosa... sobre todo de fontanería...

Lola: ¡Venga!

Pedro: Muchas gracias, ¡hasta luego!

El padre de Lola: [...] ¿Estás haciendo amigos?

Lola (en souriant): ¡Qué pesado eres! ¡Anda, vete! Que tengo que prepararme la prueba de esta tarde.

(Un paso adelante)

Tout de suite après vient le que adverbe, dont la matière sémantique est également une matière emphatique mais qui, cette fois, est nécessaire pour la bonne formation de la phrase et ne pourrait être considéré comme un effet de sens de la saisie qui précède : en effet, que vient modifier un adjectif ou un adverbe et signifie le hautdegré d'intensité de ce dernier (Moignet 1974: 226); la matière sémantique qu'il porte, même si elle est incidente à une incidence, n'en est pas moins beaucoup plus développée que dans le cas qui précède:

¡Qué bien cocinas!

¿Qué amable eres Lolita!

Poursuivons le déroulement de l'idéogénèse et nous rencontrons le que conjonctif. Ce que est à la fois intégré dans la phrase et intègre une proposition subordonnée. On voit déjà se profiler là un emploi relatif si l'on accepte de considérer que le sujet et le verbe qui introduisent que forment une sorte d'antécédent que l'on peut plus ou moins paraphraser. C'est ce que signale Le Goffic (1993: 539-540):

mais les complétives apparaissent à l'analyse comme des relatives (ou des intégratives) incomplètes, le que complétif étant un pronom neutre, attribut d'un être sous-jacent. [...] Une complétive est, en toute rigueur, un groupe pronominal : que (relatif ou intégratif) $P[e s t]$, d'où safacilité à jouer tous les rôles du nom dans la phrase.

Le que conjonctifa donc une matière sémantique ténue mais réelle de reprise d'un groupe précédent qui se développe et que l'on peut normalement paraphraser:

Pienso que deberíamos ir a tomarnos un café para despertarnos. (Mi pensamiento que...)

Me alegraría que vinieras a verme (Mi alegría que...)

Me haría mucha ilusión que publicaran el poema que has escrito (Mi ilusión que...) 
¿Quieres que nos vayamos al cine? (Tu querer que...)

Me parece que te gustará esta ciudad. (Mi parecer que...)

L'un des effets de sens à signaler est celui du que béquille du subjonctif :

¿Que Dios os cuide!

¿Que Dios os bendiga!

Nous en arrivons ensuite à un que particulier et que le français nomme vicariant (Bonnard 1981: 35; Verjans 2007). Ce que, contrairement à ce qui a pu être affirmé, est bien présent en espagnol, quoiqu'il soit particulier. En effet, il peut récupérer certaines locutions conjonctives, comme c'est le cas dans la phrase suivante :

Dado que el corpus con el que se trabajó no contiene referencias al uso del italiano, y que, por otra parte, no existe bibliografía específica acerca del contacto con el español en Chubut, no es posible por ahora profundizar en esta cuestión (Virkel: 158-159).

C'est bien une matière sémantique grammaticale complète qui est reprise dans ce cas, et nous sommes en présence, comme c'est le cas en français, d'un que qui a presque la fonction d'un pronom relatif, puisqu'il permet de récupérer une locution conjonctive (dado que) qui fonctionne en quelque sorte comme un antécédent (voir sur le sujet Thomas Verjans 2007: 147-148). Nous sommes donc dans un état subduit du pronom relatif, mais un état de l'idéogénèse plus avancé que le précédent, où la matière sémantique est plus pleine puisqu'elle récupère cette fois un véritable antécédent.

Nous en arrivons ensuite au pronom relatif dans l'idéogénèse. Cette fois, que récupère une matière notionnelle qui peut aussi bien être inanimée qu'animée et intègre cette matière dans une subordonnée.

Con Pobres gentes comienza para Dostoievski una etapa nueva en su vida. Su primera obra literaria es un éxito. Pobres gentes vincula el comienzo literario de Dostoievski a una serie de vivencias y experiencias personales de carácter burocrático de suma trascendencia para la creatividad novelística del autor. La experiencia de los estudios cursados por él en el internado y en el colegio de ingeniería militar, acentuaron el énfasis que el autor pondría en lo sucesivo en el grado o chin, como aspectos puntuales de la burocracia que tanto acapararon el interés de Dostoievski durante toda su vida (Martínez Fernández 2003: 58).

La ruptura con el ideal comunitario configura la ruptura y la tragedia vivida por el funcionario, quien desamparado, añora la vieja relación fraternal de su 
pueblo, cuya realidad ya no es capaz de articular, en parte, por la vergüenza, y en parte también, por el sentimiento de culpaque lo invade desde que es funcionario (Martínez Fernández 2003: 60).

Dostoievski es un escritor que me gusta mucho.

Les relatives périphrastiques ne sont que des effets de sens de cette saisie: lo que piensas de esta novela me interesa mucho; lo que has escrito es muy interesante.

On voit que la matière sémantique est plus pleine que dans le que vicariant et que cette matière est plus actualisée.

Une question se pose à nous pour l'étape suivante de l'idéogénèse, où l'on rencontre quédéterminant interrogatif. Pourquoi dans un tel cas pouvonsnous affirmer que la matière sémantique est plus pleine que dans le cas du pronom relatif ? C'est là un fait de présupposition : en effet, le quéinterrogatif présuppose une matière à venir alors que le que pronom relatif a besoin que la matière reprise soit déjà présente. Le contenu notionnel est donc davantage posé dans le cas où qué est déterminant interrogatif, puisqu'il présuppose un objet ou un être:

¿Qué color prefieres?

¿Qué libro has leído?

Enfin, que devient un mot complètement plein au bout de l'idéogénèse et a même la capacité de former à lui seul une phrase, comme dans cette phrase où quésignifie la surprise : Me voy a Islandia para aprender el islandés.¿Qué?

Cette phrase que nous venons de citer n'est qu'un effet de sens de cette saisie de que que l'on retrouve dans les phrases qui suivent:

Lola: Papá, ¿qué haces aquí?

El padre de Lola: Tenía una chapuzilla cerca. Bueno ¿qué?... ¿qué tal ha ido?

Lola: Pues... bien. ¡He pasado la primera prueba!

(Il serre Lola dans ses bras).

(Un paso adelante) 


\section{CONCLUSION}

Nous avons essayé de retracer dans ses grandes lignes le mouvement de l'idéogénèse du mot espagnol que. Ce mouvement est un mouvement qui actualise une matière sémantique de plus en plus développée, comme on a pu le voir. En effet, d'un que emphatique que l'on pourrait tout à fait supprimer, on passe à un que qui a pour but de signifier un haut degré ; puis l'emploi relatif est amorcé avec le que conjonctif et se poursuit avec le que vicariant et le que pronom relatif, et c'est cette fois une matière sémantique beaucoup plus développée qui se trouve présente, puisque cette matière reprend un antécédent, quelle que soit la forme de ce dernier ; enfin, que devient déterminant interrogatif qui présuppose déjà une matière à venir, et l'idéogénèse se complète par un qué pronom interrogatif qui est chargé d'une matière sémantique complète qui présuppose par lui-même l'existence d'un référent et peut former une phrase à lui seul. Nous pouvons donc proposer l'idéogénèse qui suit pour conclure:

(2) idéogénèse du mot espagnol que:

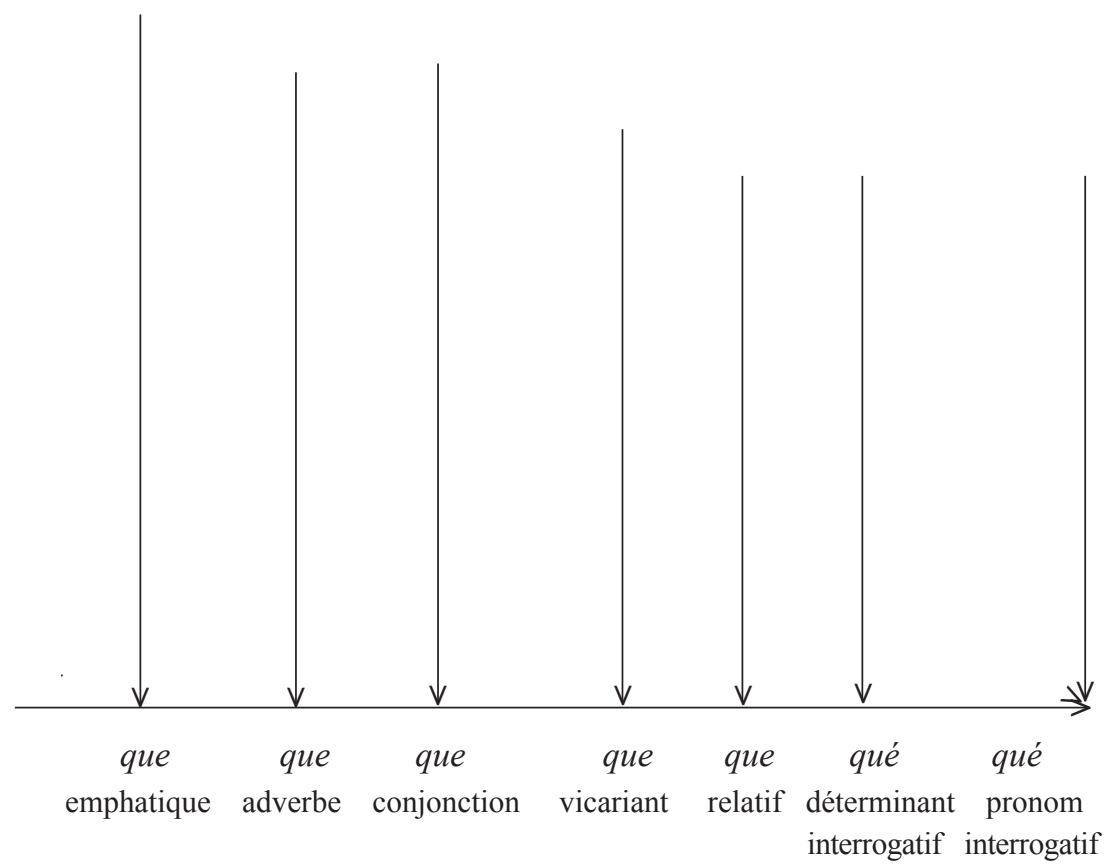




\section{REFERENCIAS BIBLIOGRAFICAS}

Bonnard, Henri. 1981.Code du français courant. Paris: Magnard.

Boone, ANNIE; ANDRÉ Joly. 1996. Dictionnaire terminologique de la systématique du langage. Paris: L'Harmattan.

Guillaume, Gustave. 1971a. Leçons de linguistique de Gustave Guillaume 1948-1949. Structure sémiologique et structure psychique de la langue française 1, publiées par Roch Valin. Québec: Les Presses de l'université Laval; Paris: Klincksieck.

1971b. Leçons de linguistique de Gustave Guillaume1948-1949. Psychosystématique du langage. Principes, méthodes et applications 1 , publiées par Roch Valin. Québec: Les Presses de l'université Laval; Paris: Klincksieck.

1973a. Principes de linguistique théorique de Gustave Guillaume. Recueil de textes inédits préparés en collaboration sous la direction de Roch Valin. Québec: Les Presses de l'université Laval; Paris: Klincksieck.

1973b. Langage et science du langage. Québec: Les Presses de l'université Laval; Paris: Klincksieck.

Le Goffic, Pierre. 1993. Grammaire de la langue française. Paris: Hachette.

Martínez Fernández, Isabel. 2003. Unas palabras sobre Pobres gentes. Eslavística Complutense 3: 57-66.

Moignet,GÉraRd. 1974. Etudes de psycho-systématique française. Paris: Klincksieck.

Soutet, Olivier. 2005. Structure bitensive de l'idéogénèse de que: soubassements théoriques et implications sémantico-syntaxiques. Cahiers de linguistique analogique 2: 277-296.

Verjans,Thomas. 2007. Sur un emploi de que: le que "vicariant". Cahiers de linguistique analogique 4: 133-155.

VIRKEL, ANA. 2005 2006. Contacto de lenguas en la etapa inicial de la colonización del Chubut (Patagonia). Anuario de lingüistica 21 22: 149-166. 